\title{
UNA PECULIAR MANIFESTACIÓN DEL PODER EPISCOPAL EN LA CATEDRAL DE LUGO. DON PEDRO LÓPEZ DE AGUIAR Y SU PROYECTO DE DIGNIFICACIÓN DE LA CAPILLA DE SANTO DOMINGO*
}

\author{
por
}

MaNuel Mosquera Agrelo

Universidad de Santiago de Compostela

\begin{abstract}
* Siglas Utilizadas en el artículo:
A.H.N.: Archivo Histórico Nacional de Madrid; A.C.L.:Archivo Catedral de Lugo; Tumbillo de Pablo Rodríguez: "Colección de los privilegios, testamentos y donaciones que contienen los monumentos del Tumbo antiguo de esta Santa Iglesia Catedral de Lugo y de los góticos originales que se ballan sueltos en el Archivo de la dicha Santa Iglesia y en los diez tomos existentes en el Archivo de la Dignidad Episcopal, copiados con la más puntual exactitud por la notoria inteligencia de el R. P. Maestro Fray Pablo Rodriguez, regente del colegio de Eslonza de la Religión de nuestro glorioso Padre San Benito en este presente año de 1763" (Archivo Catedral de Lugo); Memorias de Piñeiro: «Colección diplomática y memorias para la bistoria de la ciudad e Iglesia de Lugo. Miscelánea de varios documentos, apuntes y noticias tomadas para la bistoria de Lugo por el Dr. D. José Vicente Piñeiro y Cancio, Canónigo Doctoral, que fue de esta Santa Iglesia donde se halla» (Archivo Catedral de Lugo); Colección Diplomática de Cañizares: "Colección Diplomática de D. Buenaventura Cañizares del Rey, párroco de Lalín y canónigo de Lugo y Santiago» (Archivo Catedral de Lugo).

Agradecimientos:

El presente estudio se desarrolla en el marco de la investigación orientada a la realización de mi tesis doctoral «La conformación de los grupos sociales en el Lugo medieval: El Cabildo catedralicio como corporación institucional y grupo de poder», actualmente en realización bajo la dirección del profesor Santiago Jiménez Gómez. Manifestar mi agradecimiento a la profesora María José Portela Silva por las facilidades concedidas para la disposición de los fondos documentales de su tesis doctoral, algunos de los cuales han sido publicados recientemente. (PORTELA SIlva, María José: Colección Diplomática de la Catedral de Lugo. Santiago de Compostela, 1992). Agradecer también las absolutas facilidades concedidas por don Amador López Valcárcel, canónigo archivero del Archivo Catedral de Lugo, para la consulta de los documentos depositados en este archivo. Finalmente, manifestar mi gratitud a la Excma. Diputación Provincial de Lugo por su colaboración en la financiación de mi estancia investigadora en los archivos lucenses.
\end{abstract}


RESUMEN: En el proceso de construcción que protagoniza el obispo Pedro López de Aguiar a lo largo del siglo XIII en la diócesis lucense, destaca, por su peculiaridad, la edificación que con posterioridad será conocida como la Capilla de los Reyes de la catedral de Lugo. La interpretación tradicional transmite la idea de la colaboración real patrocinando el proceso constructivo a partir de una supuesta orden del rey Enrique II de Trastámara. Sin embargo, un pormenorizado análisis de las circunstancias que rodean este acontecimiento, parece sugerir interpretaciones bistóricas bien diferentes que nos comunican la habilidad de gobierno demostrada por el obispo López de Aguiar. Aprovechando el fallecimiento del monarca y la coronación de Juan I, consigue la confirmación de unas importantes mercedes para el sostenimiento de la corporación capitular. Logra al mismo tiempo un contacto directo entre la familia real y la catedral lucense, de lo que emana una categoría especial para la capilla de Santo Domingo - fundador de la orden a la cual pertenece el obispo- que se convierte en nueva capilla de reyes. Finalmente, en la entrada de la capilla real, figurará el escudo de la familia de Aguiar evocando la importancia de un linaje nobiliar en relación directa con el poder monárquico. En definitiva, unas circunstancias que nos comunican actuaciones de gobierno y ejercicio de poder durante la Edad Media.

Palabras clave: Capilla de los Reyes. Canonjía. Cabildo. Linaje. Monarquía. Rentas del Puerto de Viveiro. Lugo.

ABSTRACT: In the thirteenth-century program of architectural construction in the diocese of Lugo, in which Bishop Pedro López de Aguiar played the leading role, the building that would later come to be known as the royal chapel, in the Cathedral of Lugo, stands out. The traditional interpretation fosters an assumption that royal sponsorship lay behind this whole process, starting from an alleged ordinance given by King Enrique II of Trastámara. However, a detailed analysis seems to suggest a very different interpretation that reflects the bishop's own ability in government. Using to bis own advantage the monarch's death and the ensuing coronation of Juan I, be obtained the grant of several important favours (mercedes) for the support of the catbedral chapter. At the same time be succeeded in establishing a direct relationship between the royal family and the cathedral of Lugo. From this relationship a special status is awarded to the chapel of St. Dominic - founder of the order to which the bishop bimself belongs- which subsequently becomes the "royal chapel". And finally, at the entrance to the new royal chapel, the family arms of Aguiar are placed, thus evoking the importance of a noble lineage in direct relation to royal power.

KEY WORDS: Royal chapel. Cathedrals. Lugo. Nobility. Lineage. Monarchy. Viveiro.

El día 13 de Marzo de 1435 se reúnen en la sala del Tesoro de la Catedral de Lugo las máximas dignidades de la diócesis en ese momento. Al encontrarse vacante la sede episcopal, a pesar de que el nombramiento del nuevo obispo ya se había producido, la representación del prelado la realizan el Deán D. Martín Vidal de Santiago y los Arcedianos de Neira y de Dozón, ejerciendo como vica-

Hispania, LXI/2, núm. 208 (2001) 475-492 
rios episcopales. Completan el grupo capitular el Chantre y diversos canónigos. El notario Pedro Fernández de Silvarrey - cuyo trabajo podemos documentar entre los años 1414 a 1438 - ejecuta la importante tarea de fijar por escrito lo que acontece en esta reunión. En ese momento se presenta en el Tesoro el canónigo y beneficiado Pedro López de Aguiar, el cual, mediante la documentación que adjunta, solicita que el Cabildo le reconozca su capacidad para nombrar a un nuevo capellán y canónigo.

Tan sólo esta escena que acabamos de describir basta por sí sola para justificar la redacción de este trabajo: Ni el obispo, ni el propio cabildo nombran al candidato para la canonjía vacante. Es uno de los canónigos el que reclama y consigue que se reconozca su derecho a nombrar a un nuevo miembro de la corporación capitular, (hecho que, por otra parte, caracteriza este proceso que posteriormente se institucionalizará con la figura de las capellanías colativas de sangre).

Lo que se presenta como un hecho peculiar dentro de lo que el Derecho Canónico o los Estatutos Capitulares determinan para el nombramiento de los nuevos miembros del cabildo, tiene su explicación en lo acontecido medio siglo antes. Estos sucesos y las características que rodean esta particular canonjía capellanía será el objeto de nuestra atención en el presente artículo, intentando trazar unas líneas directrices que nos ilustren sobre comportamientos generales del Cabildo Catedral de Lugo. Esto se establecerá, sobre todo, en lo referente a sus relaciones con la cátedra episcopal para el nombramiento, ceremonias, rentas, derechos y obligaciones de sus canónigos a lo largo de la Baja Edad Media.

\section{LOS OBJETIVOS DE ESTE TRABAJO}

Diversos acontecimientos que conforman la peculiaridad de la capellaníacanonjía de Santo Domingo de los Reyes en la catedral de Lugo, incitan a reflexionar sobre la destacada habilidad de gobierno que caracterizaba la figura del obispo don Pedro López de Aguiar.

Para intentar una aproximación más completa y detallada a estas actuaciones de poder, centraremos nuestra atención en un proceso sutil acontecido en la catedral de Lugo entre los años 1379 y 1381.

Podremos observar así como don Pedro de Aguiar consigue la constitución de la capilla de Santo Domingo de Guzmán - que el propio obispo acababa de construir-, en capilla real de la catedral lucense, pero ningún indicio documental nos muestra que los monarcas hayan participado en la construcción y patrocinio de la capilla, excepto una confirmación hecha por Juan I en el año 1381 en la que menciona una orden real para la construcción de la capilla emanada de su padre, Enrique II.

A lo largo de este trabajo trataremos de matizar este supuesto patrocinio, proponiendo otras interpretaciones y explicaciones de los motivos que, en su momento, pudieron inducir al obispo Aguiar a apreciar la transcendencia de

Hispania, LXI/2, núm. 208 (2001) 475-492 
establecer una relación directa entre el cabildo, la catedral de Lugo y la dinastía de los Trastámara.

\section{DESCRIPCIÓN DE LAS FUENTES DOCUMENTALES}

La base documental que fundamenta el conocimiento de los hechos que se analizan en este artículo, se estructura sobre el contenido de un acta notarial de principios del siglo XV, concretamente del año 1435. Se encuentra en la actualidad depositada en el Archivo Histórico Nacional de Madrid catalogada como documento número 12 de la carpeta 1332-G de la Sección de Clero del mencionado archivo. Se trata de un pergamino original en el que se recoge un acta notarial en gallego y con letra cortesana. De este documento se conservan también varias copias en el Archivo de la Catedral de Lugo, tanto en el llamado "tumbillo nuevo" de fray Pablo Rodríguez ${ }^{1}$ como en las colecciones diplomáticas de José V. Piñeiro ${ }^{2}$ o de don Buenaventura Cañizares del Rey 3 .

Tal como ha sido mencionado al principio de este trabajo, la acción fundamental narrada en este documento se centra en la fecha del 13 de marzo de 1435, momento en el que Pedro López, sobrino del obispo don Pedro López de Aguiar - fundador de la capilla de Santo Domingo-, solicita del cabildo el nombramiento del nuevo capellán-canónigo de la capilla de Santo Domingo de los Reyes, aportando los documentos que justificaban la autoridad en la que fundamentaba la potestad para tal ejercicio.

Estos dos documentos aportados por el beneficiado Pero Lopes quedaron recogidos en el texto del acta redactada en 1435. El primero de ellos, datado el 1 de julio de 1379, especifica los términos de los acuerdos estipulados entre el cabildo de Lugo y el obispo don Pedro López de Aguiar en referencia a la capilla de Santo Domingo. En el segundo de los documentos, datado en el 8 de septiembre de 1388 , se plasman de nuevo una serie de estipulaciones tratadas entre el obispo López de Aguiar y el cabildo. Estas nuevas condiciones, indican una clara reorganización administrativa de los bienes dependientes de la capilla de Santo Domingo, acuerdos que, comparados con los términos alcanzados en la reunión del año 1379, nos proporcionarán los indicios indispensables para fundamentar hipótesis y alcanzar las conclusiones que se han planteado como objetivo de este trabajo.

Son, además, estos tres documentos unidos en uno, los que constituyen esta acta notarial como la única muestra de nombramiento y colación de un cargo capitular lucense en la Edad Media, una tipología inexistente en el corpus documental de la catedral de Lugo sobre todo debido a los diversos incendios que

A. C. L., Tumbillo de Pablo Rodríguez, fol. 148r. a 158v.

A. C. L., Memorias de Piñeiro, vol. IV, fol. 35v. a 41 r.

3 A. C. L., Colección Diplomática de Cañizares, doc. nº.572, vol. IV, pp. 1154 a 1162.

Hispania, LXI/2, núm. 208 (2001) 475-492 
afectaron al fondo documental catedralicio. Por estas circunstancias, los hechos narrados en el acta notarial de 1435 proporcionan una riqueza de datos que resultan de gran trascendencia, tanto para el conocimiento de las circunstancias concretas de la capilla de Santo Domingo, como para su generalización a otros aspectos del funcionamiento administrativo del cabildo lucense a lo largo de la Edad Media.

Por otra parte, las informaciones extraídas de este documento, tienen que ser completadas y contrastadas con otros textos del medioevo lucense. Así, resulta fundamental la lectura de aquellas fuentes documentales en las que encontramos noticias de la intención del obispo don Pedro de Aguiar para ejecutar la construcción, en la propia catedral, de una capilla dedicada a Santo Domingo, fundador de la orden religiosa a la que pertenece el obispo. Los acuerdos establecidos entre el obispo y el escudero Gómez Aras de Pallares el 4 de enero de 1357, así como el testamento de éste datado el 2 de agosto de $1360^{4}$, proporcionan claridad sobre la financiación del proceso de construcción y del mantenimiento de la capilla catedralicia que estamos estudiando.

Información fundamental para nuestro estudio es aquella que se desprende de la consulta de los privilegios emanados de la cancillería real. Interesa cualquier dato que notifique confirmaciones, privilegios y mercedes concedidas por Pedro I, Enrique II y Juan I, particularmente si afectan a aspectos relacionados históricamente con la capilla de Santo Domingo, tales como, por ejemplo, la donación de una cantidad de las rentas del puerto de Viveiro a la catedral lucense. Pero, sobre todo, el "albalá" real de Juan I del 17 de abril de 1380 en el que confirma la orden real de su padre para proceder con la construcción de la capilla de los Reyes, es objeto preferente de nuestra consulta y se constituye en la pieza clave de la documentación real para la realización de este trabajo.

Finalmente, documentos como el pleito establecido entre el obispo don Pedro López y Leonor Rodríguez ${ }^{5}$, segunda mujer de Gómez Aras de Pallares, por la disposición de las rentas donadas por el escudero, así como aquellas noticias referentes a los bienes de la capilla de Santo Domingo, contribuyen en el desarrollo de las hipótesis y en la formulación de las conclusiones del estudio ${ }^{6}$.

\footnotetext{
4 Manso Porto, Carmen.: Arte Gótico en Galicia: Los Dominicos. La Coruña, Fundación Pedro Barrié De La Maza, 1993.

5 A. C. L., Estante 3, Legajo 5, $\mathbf{n}^{\circ} .35$

6 El carácter de esta investigación motiva que la consulta y las referencias vinculadas a las fuentes documentales inéditas prevalezcan sobre estudios y obras ya publicadas. Sin embargo, se citan a continuación algunas de las publicaciones que pueden contribuir a la contextualización bibliográfica de este artículo.

Sobre los aspectos más directamente relacionados con la figura del obispo Aguiar y de la Capilla de los Reyes consúltense los siguientes trabajos: PARDO VILLAR, Antonio: «Dominicos lucenses ilustres. El obispo Fray Pedro López de Aguiar» en Boletín de la comisión provincial de monumentos bistóricos y artísticos de Lugo (Lugo), vol. I, nº 1 (1943), pp. 113-114. MANSO PORTO, Carmen: «El obispo fray Pedro López de Aguiar, O.P. (1349-1390). Reseña biográfica y aproximación a los principales acontecimientos en su diócesis durante el reinado de Pedro I» en Archivo Dominicano, vol.
} 


\section{LA CAPILla y CAPELlanía-CANONJía DE SANTO DOMINGO DE LOS REYES}

\subsection{La Capilla de Santo Domingo}

La capilla de Santo Domingo ${ }^{7}$, también denominada en la documentación, a partir del año 1383, como "Capela de San Domingo dos Reys» o "Capela Nova dos Reys», protagoniza una de las múltiples actividades arquitectónicas que acontecen en el Lugo de este periodo cronológico, siendo la primera edificación que se efectúa en el exterior de la antigua catedral románica, adosada al lateral norte y aprovechando el ángulo al que da lugar el cruce de la nave longitudinal y el brazo del crucero.

La primera noticia que se puede documentar en referencia a la intención de López de Aguiar para proceder con la edificación de una capilla en la catedral de Lugo y disponerla bajo la advocación de Santo Domingo podemos constatarla a partir de los documentos protagonizados por el escudero Gómez Aras de Pallares. Tanto en 1357, como en su testamento de 1360, Gómez Aras dispone bajo

XIV (1993), pp.43-67. Id.: Arte Gótico en Galicia: Los Dominicos. La Coruña, Fundación Pedro Barrié De La Maza, 1993. Mosquera AGrelo, Manuel: «La Capellanía-Canonjía de Santo Domingo de los Reyes en la Catedral de Lugo. Datos para el conocimiento del Cabildo lucense medieval» en Lucensia (Lugo), vol. IX, nº 18 (1999), pp. 79-97. García CONDE, Antonio - López VALCÁrCel, Amador: «Episcopologio lucense (X - 1900)» en Liceo Franciscano (Santiago de Compostela), Año XLIII - $2^{a}$ época (Enero - Diciembre 1991). Números 130 - 132.

Para la contextualización y ampliación de datos sobre la ciudad de Lugo en la época en la que se centra el presente estudio examínense las obras siguientes: PORTELA Silva, María José - GARCía Oro, José: «La Iglesia y la ciudad de Lugo en la Baja Edad Media. Los señoríos, las instituciones, los hombres» en Cuadernos de Estudios Gallegos (Santiago de Compostela), Anexo nº 24 (1997). García Oro, José: «La ciudad de Lugo y la Iglesia en la Baja Edad Media» en Lucensia (Lugo), nº 3 (1991), pp. 4780. Id.: «La nobleza gallega en la Edad Media. Las casas nobles y sus relaciones estamentales», en Liceo Franciscano (Santiago de Compostela), Años XXXIII-XXXIV (Enero - Diciembre 1980-81). Números 97 - 102. Id.:Galicia en los siglos XIV-XV, Coruña 1987: Colección Galicia Histórica.

Finalmente, atendiendo al período cronológico del artículo y a su vinculación con los conflictos de mediados del siglo XIV deben tenerse en cuenta obras de carácter más ámplio que relacionan este trabajo con el ámbito hispánico y la dinastía Trastámara: NiETo SORIA, José Manuel: «La configuración eclesiástica de la realeza trastámara en Castilla (1369-1474). Una perspectiva de análisis» En la España Medieval, 13, León 1990. VALDEÓN BARUQue, Julio: Enrique II de Castilla: la guerra civil y la consolidación del régimen (1366-1371), Valladolid 1966. Id.: «Notas sobre las mercedes de Enrique II de Castilla» en Hispania, $\mathrm{n}^{\circ} .108$ (1968). Id.: «Las sociedades urbanas en la guerra civil de Castilla de mediados del s. XIV» en Mayurqa. Homenatge a Alvaro Santamaría, Palma de Mallorca 1989. Id.: «Enrique II (1369-1379)», en Corona de España VII - Reyes de Castilla y León, Palencia 1996.

7 Para obtener una información más completa en lo referente a la fundación, localización, aspectos organizativos y los diversos temas de relación entre la capilla de Santo Domingo y el Cabildo de Lugo, consúltese: MosQuera Agrelo, Manuel: «La Capellanía - Canonjía de Santo Domingo de los Reyes en la Catedral de Lugo. Datos para el conocimiento del Cabildo lucense medieval» en Lucensia (Lugo), vol. IX, $\mathrm{n}^{\circ}$ 18, (1999), pp. 79 - 97.

Hispania, LXI/2, núm. 208 (2001) 475-492 
la responsabilidad del obispo una completa serie de bienes manifestando ser donados con el objetivo de construir una capilla en la iglesia catedral de Lugo ${ }^{8}$.

En el año 1379 conocemos el nombre del primer capellán y se constata, por tanto, la finalización de su construcción. A partir de ese año podemos documentar diversos personajes que se encargan del cuidado de la capilla y otros múltiples aspectos que serán objeto de tratamiento a lo largo del artículo.

Finalmente, la capilla construida bajo la dirección del obispo López de Aguiar, dejó de existir con entidad propia cuando en el año 1611 quedó unida a la que entonces se denominaba «capilla de San Froilán» - que fuera construida bajo el mecenazgo de don Gómez García de Gayoso-. En ese momento la unión de ambas capillas acaba por constituir la que hoy en día se conoce como «capilla del Pilar».

\subsection{Capellanía y Canonjía}

El origen de las peculiares circunstancias que rodean la reunión y nombramiento de 1435, comentadas desde las primeras líneas de este trabajo, se encuentra en los acuerdos determinados en el año 1379 entre el obispo don fray Pedro López y el Cabildo de Lugo.

Tal como sucede en el resto de las capillas de la catedral, la capilla de Santo Domingo queda, una vez finalizada su construcción, al cuidado de un capellán, siendo Diego López - en el año 1379 - el primero del que se tiene noticia9.

Sin embargo, muy pronto procedió don Pedro a cambiar las características de su capilla. Así, en el ya referido documento de 1 de julio de 1379, fray Pedro López y su Cabildo acuerdan conceder a perpetuidad a la persona encargada de la capilla de Santo Domingo el privilegio de ser nombrado canónigo, entrando a formar parte del cabildo con todos los deberes y derechos inherentes a su condición canonical.

8 «Sabeam quantos esta carta virem commo eu Gomes Aras de Pallares, fillo que fuy de Andreu Aras, outorgo et connosco que dou en pura doaçon por remedio de minna alma et de Maria Affonso, minna muller que fuy, et de aquelles a que soo tiudo, a a iglesia de Lugo para huna cappella conven a saber[...]", Madrid, A.H.N., Códice 417 B, folio 55 r. y v.

"Item mando aa cappilla que han de faser enna Iglesia de Lugo, seis casares de herdade para que digan y huna missa cada dia por la miña alma e a da dita Lionor Rodrigues e da dita Moor Afonso" Testamento de Gómez Aras de Pallares editado por Carmen Manso (MANSo PorTo, Carmen: opus cit.)

9 «Et esta aneyxaçon sobredita das ditas coensia et sua prebenda con todos seus dereitos sobreditos et con outros quaesquer que lle perteescan fazemos commo dito he de consintemento dos ditos dean et cabidoo a a dita capela por tal condiçon que Diego Lopes, capelan da capela, et proveytos et dereituras quaesquer segundo que de suso son declarados das ditas coensia et sua prebenda con todos seus dereitos sobreditos et con outros quaesquer segundo que de suso son declarados das ditas coensia et sua prebenda». Madrid, A.H.N., Sección Clero (Lugo-Cat.), Carp. 1332 G, doc. $n^{\circ} 12$. 
Este privilegio podría concederse creando una nueva canonjía, ya que el único requisito sería el contar con las suficientes rentas que asegurasen la dignidad de la nueva prebenda. Sin embargo, el fallecimiento de uno de los canónigos lucenses, Alfonso López, favorece la intención del obispo Aguiar, y le permite, con la aceptación capitular, asociar esta canonjía vacante al cargo de capellán de la capilla construida con su financiación.

Vistos los hechos, no resulta difícil imaginarse los motivos que impulsan a don Pedro en estos acuerdos, intentando conseguir sobre todo un estatuto de especial importancia para la capilla dedicada a Santo Domingo de Guzmán, fundador de los dominicos, la orden a la que él pertenece. Por último, pero no por ello menos importante, don Pedro no olvida su condición de primogénito de la noble familia de los Aguiar $^{10}$. Este acuerdo nos indica claramente algunas de las pretensiones del obispo. Ha costeado la construcción de una capilla en la catedral y ha establecido lo necesario para asegurar su mantenimiento futuro al constituir una prebenda con una importante dotación creada a partir de generosas donaciones y parte de su patrimonio personal, destinada a la Mesa Capitular.

No está, por tanto, fuera de lugar que, ya que todo ello se ha constituido, en parte, gracias a la generosidad de la familia de los Aguiar y de distintos individuos relacionados con ella, sean los miembros futuros de este linaje los que se encarguen de designar la persona que consideran idónea para ejecutar el cuidado y administración de lo que don Pedro ha creado. Más justificado aún si tenemos presente la enorme influencia que este linaje debe tener en la estructura del cabildo catedral de esta época al dictar el obispo que la designación provenga en primer lugar de aquel familiar que fuese beneficiado en el Cabildo lucense. Se crea así un puente no sólo hacia la patrimonialización del cargo, sino que, al mismo tiempo, también se nos muestra un grupo de poder interno en el Cabildo, que, con su influencia, establece de cara al futuro una manifiesta injerencia, incluso civil ${ }^{11}$, en un cargo eclesiástico. Esta capacidad de nombramiento, implicaba, desde el propio momento de la concesión de este privilegio, una importante influencia ante cualquier acuerdo capitular, al contar el linaje Aguiar, de antemano, con un canónigo que, con derecho a voz y voto en la reunión, iba a mostrarse absolutamente favorable a los intereses de aquella familia a la que le debía el puesto en el Cabildo. Tal vez, como contrapunto final del epígrafe, convenga matizar la trascendencia de lo que, aunque aquí

10 «El pontificado más duradero de los obispos mendicantes gallegos fue sin duda el de fray Pedro López de Aguiar (1349-1390). En la diócesis lucense realizó una importante gestión, que pudo reforzar por su condición de primogénito de la Casa de Aguiar -Lugo-». MANSO PORTO, C.: opus cit. Tomo I, p. 39.

11 «Et non avendo enna dita nosa iglesia benefiçiado de noso linageen quando acontesçe de se finar aquel que for coengo et capelan, que o mayor et mellor de noso linagen posa presentar clerigo de missa ou que posa logo ordenar de misa, que aia a dita coensia et prebenda dela, et que seia capelan et servidor da dita capela con as condiçoes que ditas son». Madrid, A.H.N., Sección Clero (Lugo-Cat.), Carp. $1332 \mathrm{G}$, doc. $\mathrm{n}^{\circ} 12$.

Hispania, LXI/2, núm. 208 (2001) 475-492 
parece un hecho singular, a partir de estos momentos será algo institucionalizado en la Iglesia peninsular y conocido como la fundación de las Capellanías Colativas de Sangre.

\subsection{Las Obligaciones litúrgicas del capellán y del cabildo}

No podemos perder de vista que desde el momento que la canonjía queda anexada a la capilla, el capellán adquiere también una serie de obligaciones que se añaden a las que ya eran inherentes a su cargo. La generosa cantidad de bienes que fundamentan la existencia del cargo de capellán de Santo Domingo, conlleva una serie de obligaciones que se fijan de forma estricta y concreta en los diversos acuerdos establecidos entre el obispo y el cabildo.

Concretamente, un primer conjunto de exigencias se centra en las obligaciones litúrgicas, dato de trascendencia de cara a las posteriores indagaciones sobre el protagonismo de los monarcas en el mecenazgo de esta capilla: el capellán deberá oficiar en la capilla de Santo Domingo una misa para alabanza de Dios y de Santo Domingo. Debe tener siempre presentes las almas de aquellos que con sus donaciones contribuyeron en la constitución de la capellanía que lleva anexa la canonjía y prebenda que el oficiante detenta, especificando concretamente los nombres del arcediano de Deza, don Alfonso Gómez, del escudero Gomes Ares de Pallares, y de su primera mujer, Mayor Afonso. Por último, añade también al canónigo Fernan Afonso ${ }^{12}$.

Se fija una periodicidad diaria para la celebración eucarística que tan sólo puede ser oficiada por el propio capellán a no ser que haya alguna dificultad o traba que se lo impida. De existir este impedimento, se le concede permiso al capellán para que pueda nombrar a un sustituto que reúna las cualidades idóneas para este oficio.

Para conseguir una mayor pulcritud y dignidad litúrgica, don Pedro reclama la colaboración capitular. Dispone una serie de estipulaciones litúrgicas que implican la participación del Cabildo en el culto de la capilla. Concretamente, se encarga de recordar al Cabildo las obligaciones que tienen con las capillas catedralicias $^{13}$, lo que conlleva, sobre todo, una serie de actos en honor del santo patrono de la capilla, implicando la realización de una procesión y el rezo de

12 «et que o dito Diego López et depoys del os outros capelaes que por tempo foren da dita capela cada hun en seu tempo suçesive han de diser enna dita capela para senpre sua misa a [loor] de Deus et de San Domingo, et por nosa alma [et por las de noso] padre et madre et avoos, et dos ditos arçidiago et Gomes Ares, et Mayor Afonso, et Fernan Afonso, aprovandoos os ditos dean et cabidoo». Madrid, A.H.N., Sección Clero (Lugo-Cat.), Carp. 1332 G, doc. nº 12.

13 «Outrosy ordenamos que o dito dayan et cabidoo vaan en proçison a dita capela enno dia das vesperas da vegilia de San Domingo do mes de agosto senpre, segundo que van a a outras capelas da dita iglesia por la vespera et dia de vocaçon delas». Madrid, A.H.N., Sección Clero (LugoCat.), Carp. $1332 \mathrm{G}$, doc. $\mathrm{n}^{\circ} 12$. 
maitines en la víspera de la vigilia de Santo Domingo, así como una misa cantada el propio día del patrón. Para completar esta labor de engrandecimiento del culto, compensa a la Mesa Capitular con una importante donación ${ }^{14}$, consiguiendo el prelado una activa participación del clero capitular en la liturgia de su capilla que incluye una solemne celebración eucarística a oficiar cada sábado, en la que deben resplandecer algunos de los más destacados ornamentos litúrgicos de la época, como son las valiosas capas que el Cabildo posee, procediendo después al reparto de las preceptivas rentas entre los asistentes al acto.

\subsection{La cuestión económica}

Analizadas las obligaciones, se planteará en este epígrafe un acercamiento a las compensaciones, al análisis de las rentas que sostienen la canonjía que venimos estudiando. ¿Cuáles son estas rentas?, ¿Cuál es su origen?, ¿A quién y para qué serán destinadas?. Estas son algunas de las preguntas que nos permitirán un primer acercamiento a algunos aspectos económicos de una canonjía lucense bajomedieval.

Contamos en nuestro caso particular con dos conjuntos de rentas absolutamente diferenciados que van a confluir en la persona que ocupe el cargo de capellán. De un lado, un grupo de rentas directamente relacionadas con la condición de canónigo, rentas que ineludiblemente iban adscritas a la canonjía que ahora se anexa a la capilla:

«por ende nos aprovandoo os ditos dean et cabidoo aneixamos as ditas coensia et sua prebenda con todos seus froytos et rendas et proveitos et con todos comprimento de dereito canonico et çivil a elas perteesçentes en futuro et en presente (...) aa capela chamada de San Domingo que nos edificamos et fezemos ${ }^{15_{\nu}}$.

Estas rentas provienen de los beneficios obtenidos a partir de la administración de las propiedades capitulares, de los repartos efectuados por la asistencia a los cultos, así como del cobro de las correspondientes partes que cada canónigo percibía de los distintos ingresos adscritos a la mesa capitular.

No obstante, podemos apuntar una situación de crisis que afectaba a las rentas capitulares en este siglo. Es por ello por lo que don Pedro decide además disponer un importante grupo de rentas que directamente redundaría en una

14 «Outrosy nos, o dito sennor obispo, con outorgamento dos ditos dayan et cabidoo anexamos logo et iuntamos a a dita mesa do dito cabidoo a metade da iglesia de Santo Andre de Ribeira de Minno, et a metade da iglesia de Santa Maria de Ferreira sen cura, tanto que elas et cada huna delas vaguen por aqueles que as ora teen, con tal condiçon que os ditos dean et cabidoo et seus suçesores sian et leven os dizemos et rendas et froytos et dereytos que perteesçen a os quinoons destas iglesias». Madrid, A.H.N., Sección Clero (Lugo-Cat.), Carp. 1332 G, doc. $\mathrm{n}^{\circ} 12$.

15 Madrid, A.H.N., Sección Clero (Lugo-Cat.), Carp. 1332 G, doc. $\mathrm{n}^{\circ} 12$.

Hispania, LXI/2, núm. 208 (2001) 475-492 
mejor situación económica del rector de su capilla y que dinamizasen y mejorasen indirectamente la situación económica de la mesa capitular. Un conjunto de valores que agrupa 12 casales (casares de erdade), 8 viñas, 7 Leiras, 1 leiro, 1 cortiña, 1 huerto y varias casas —en uso y abandonadas - en diversas calles de la ciudad de Lugo. Propiedades cuya forma de adquisición podemos documentar tanto a través del acta notarial que manejamos como por otros testimonios de la documentación lucense.

Así un primer lote de bienes proviene de la donación del escudero Gómez Aras de Pallares y de su primer matrimonio con Mayor Afonso. Casi la mitad de los casales, junto con varias casas, dos leiras, una viña y la parte que le pertenecía en el "Paaço de Malle» se encuentran documentados en el texto del día 4 de enero de 1357 por el que el escudero hace al Cabildo «doaçon por remedio de minna alma et de Maria Affonso, minna muller que fuy (...) a a iglesia de Lugo para huna cappella ${ }^{16}$ ». En la misma línea, encontramos una nueva referencia a su mecenazgo para con la capilla construida por don Pedro de Aguiar, en el testamento del propio Gómez Aras de Pallares del 2 de Agosto de $1360^{17}$.

En segundo lugar, un importante conjunto de posesiones se deben a los bienes legados por el Arcediano de Deza don Alfonso Gómez, cuya presencia como clérigo del Cabildo lucense podemos atestiguar entre los años 1320 a 1364.

El conjunto patrimonial se completaría con el legado del canónigo Fernando Afonso (que aparece como miembro del Cabildo entre los años $1300 \mathrm{y}$ 1349) y aquellas pertenencias particulares adquiridas por el propio obispo.

Podemos entender, por lo tanto, que don Pedro ha conjuntado un patrimonio concreto que cede en "préstamo" a su capilla y a su capellán, patrimonio que irá parejo a la canonjía y que dependerá de la mesa capitular mientras los futuros obispos y capitulares consientan en cumplir los acuerdos tratados en 1379. De no ser así, las cláusulas del pacto de 1379 son taxativas, al dejar claro que las propiedades se entregan para el encargado de la capilla, y nunca a la canonjía ${ }^{18}$.

16 Madrid, AHN, Códice 417 B, folio 55 r.-v.

17 «Item mando aa cappilla que han de faser enna Iglesia de Lugo, seis casares de herdade pra que digan y huna missa cada dia por la miña alma et a da dita Lionor Rodrigues e da dita <Moor $>$ Afonso». Transcripción documental tomada de: MANSO PORTO, C.: opus cit.

18 «et que o dayan et cabidoo dela seian tiudos de o resçeber entresy enna dita iglesia por seu coengo et companeiro, et que asi seia proviudo da dita coensia et de sua prebenda para senpre (...) et que os dayan et cabidoo da dita iglesia seian tiudos et obligados de as faser asi sanna et de paz con todos seus dereytos sobreditos a os ditos capelaes da dita capela suçesivemente, et non las fazendo asi saas et de pas con todos seus dereytos que se tornen todos los erdamentos et casas et beens sobreditos a a dita capela et a os capelaes dela suçesive». Madrid, A.H.N., Sección Clero (LugoCat.), Carp. 1332 G, doc. $\mathrm{n}^{\circ} 12$. 


\subsection{Las transformaciones de 1388}

Apreciamos la importancia de todo el conjunto de pertenencias que acabamos de pormenorizar, pero desconocemos el montante económico que éstas podían suponer en rentas. Sin embargo, a tenor de lo que observamos en el traslado notarial de 1421 (con respecto a la reunión efectuada el 8 de setiembre de 1388) podemos intuir la trascendencia que estas rentas tenían para la mesa capitular.

La explicación de lo acontecido en esta reunión capitular resulta compleja. Precisamente esta interpretación aparenta ser complicada porque desconocemos el motivo que origina lo que aquí se está acordando. ¿Por qué razón, cuando aún no han pasado diez años desde los acuerdos de anexión entre obispo y Cabildo, las dos entidades se ven obligadas a clarificar de nuevo las condiciones de unas rentas que ahora son donadas por el prelado a la Mesa Capitular? ¿Cuál es el factor determinante del serio compromiso adquirido por el Cabildo al comprometer todos los bienes de su administración del mes de enero como fianza por la recepción de las rentas de la capilla que nos ocupa?.

Estos bienes ya parecían tener un destino nítidamente estipulado en los compromisos adquiridos en 1379. Aunque no contemos entre los pergaminos originales de la documentación lucense con la mención concreta del determinante de este proceso, podemos apuntar algunas impresiones. Analicemos esta sección del documento desde su inicio.

El 8 de setiembre de 1388 se celebra un cabildo en el coro de la catedral. Los capitulares presentes son prácticamente los mismos que figuran en la anexión de 1379. Entre las dignidades sólo cabe destacar la novedad de D. Gonzalo Ozores sustituyendo a D. Pedro Ares al frente del arcedianato de Triacastela. Desde la primera línea se observa la firme intención de comprometerse a cumplir todas las estipulaciones que ya conocemos con respecto a las peculiares condiciones de la canonjía anexada a la capilla de Santo Domingo ${ }^{19}$, sobre todo en lo que respecta a la admisión dentro del cabildo de la persona que fuese designada como capellán ${ }^{20}$, manifestando reiteradamente su intención de no denunciar o intentar anular los acuerdos prefijados y renunciando

\footnotetext{
19 «prometemos et outorgamos por firme et solemne stipulacion por nos et por nosos suçesores de atender et gardar et conprir et fazer todas las cousas et cada huna delas scriptas et contiudas en hun instromento de anexaçon de huna coengia et sua vagante». Madrid, A.H.N., Sección Clero (Lugo-Cat.), Carp. 1332 G, doc. n 12 (Traslado notarial de 1421-10-01).

20 «et obligamos firmemente por nos et por nosos suçesores para senpre jamays de faser ordenar et procurar et tractar que qualquer que for [promovido] por capelan a dita capela, segundo he contiudo enno dito instromento segundo a ordenaçon que o dito senor obispo sobrelo ordenou, ou ordenar, que seia coengo desta iglesia, et noso yrmao et companeyro et que lle seia asignado [...] enno dito cabidoo, et escalo enno coro cum plenitudine iuris canonici et que aia huna prebenda entregamente, segundo cada hun dos outros coengos, segundo as condiçoes et modos enno dito instromento contiudos". Madrid, A.H.N., Sección Clero (Lugo-Cat.), Carp. 1332 G, doc. $\mathrm{n}^{\circ} 12$ (Traslado notarial de 1421-10-01).
} 
explícitamente a todo tipo de derechos que les amparasen al respecto ${ }^{21}$. Resulta sorprendente este inaudito empeño del Cabildo por confirmar de forma tan insistente algo que ya se había acordado previamente. No obstante estos no son los únicos aspectos extraños que se desarrollan en el tenor documental del traslado notarial.

En medio del compromiso de aceptación del capellán como canónigo, y enumerando sus derechos nos encontramos la siguiente frase: «et que aia huna prebenda entregamente, segundo cada hun dos outros coengos... ${ }^{22}$ ». Posteriormente, entre la noticia de la provisión de un nuevo capellán-canónigo se confirma lo que acabamos de transcribir: «que o posese enno numero dos coengos et ho contase enna dita coensia et prebenda dela segundo os outros coengos ${ }^{23}$ ». El rico patrimonio fijado por el obispo parece quedar ahora al margen de la administración de la capilla, disfrutando el capellán-canónigo de una prebenda igual a la del resto de los canónigos. Por otra parte los capitulares se esfuerzan en dejar patente que las rentas de ese «desaparecido patrimonio» pertenecen indiscutiblemente a la Mesa Capitular ${ }^{24}$. Es más, la única condición que, sobre la base del documento, podría justificar que la capilla de Santo Domingo volviese a disfrutar de todas las rentas estipuladas en 1379 , se produciría si en el futuro se negase al capellán la posibilidad de adquirir la condición de canónigo, o, lo que es lo mismo, incumpliendo las condiciones fundamentales de anexión de la canonjía a la capilla ${ }^{25}$.

Para enmarañar más este entramado, distinguimos en el documento claros indicios de que la Mesa Capitular contempla abiertamente la posibilidad de hacer plena utilización de esas rentas, situación que el Cabildo se compromete

21 «et para non poder vinir nen pasar contra elas por nos nen por outro en juyso nen fora del en algun tempo expresamente renunçiamos a todo dereyto canonico et çivil scripto et non scripto, [uso] ou custume et pribilegio et constituyçon et benefiçio da ley ou [...] et a todo dereyto ou auçon porque nos et nosos subçesores podesemos vinir et pasar contra esto que de suso dito he, ou contra parte delo, et todo ho tiramos et partimos de nos et de nosos suçesores para senpre jamays». Madrid, A.H.N., Sección Clero (Lugo-Cat.), Carp. 1332 G, doc. $\mathrm{n}^{\circ} 12$ (Traslado notarial de 1421-10-01).

22 Madrid, A.H.N., Sección Clero (Lugo-Cat.), Carp. 1332 G, doc. $\mathrm{n}^{\circ} 12$ (Traslado notarial de 1421-10-01).

23 Madrid, A.H.N., Sección Clero (Lugo-Cat.), Carp. 1332 G, doc. $\mathrm{n}^{\circ} 12$ (Traslado notarial de 1421-10-01).

24 «todos los ditos herdamentos et casas et beens contiudos enno dito instromento, que o dito sennor obispo anesou a a dita nosa mesa». Madrid, A.H.N., Sección Clero (Lugo-Cat.), Carp. 1332 $\mathrm{G}$, doc. $\mathrm{n}^{\circ} 12$ (Traslado notarial de 1421-10-01).

25 «et non agardando, nen atendendo, nen comprindo as ditas clausulas et condiçoes et cada huna delas contiudas enno dito[instromento], ou [indo] et pasando contra elas en alguna maneyra et por qualquer rason prometemos et outorgamos por nos et por nosos suçesores de dar et tornar et entregar a a dita capela et a o capelan, que en ela for en nome deles, todos los ditos herdamentos et casas et beens contiudos enno dito instromento (...) libres et quitos et desenbargados tan ben provados et reparados commo agora estan». Madrid, A.H.N., Sección Clero (Lugo-Cat.), Carp. 1332 $\mathrm{G}$, doc. $\mathrm{n}^{\circ} 12$ (Traslado notarial de 1421-10-01). 
a solucionar en el improbable caso de que estas rentas tuviesen que ser devueltas a la capilla ${ }^{26}$. Incluso en el caso de que no pudiesen cumplir esta condición, establecen en concepto de "fianza" todos los bienes de una de las administraciones del Cabildo para cubrir los daños que pudiesen causarse al patrimonio de la capilla ${ }^{27}$.

\subsection{Indicios de un sutil acto de gobierno}

Algo ha acontecido en los años que transcurren entre 1379 y 1388 para que el obispo don Pedro acepte abiertamente que el Cabildo disponga de unas rentas destinadas específicamente a su capilla y que tantos años tardó en reunir. Son varias las hipótesis que se nos ofrecen a la hora de encontrar explicación a este cambio de actitud.

Posibilidad a tener presente sería la que se desprende de que el capellán tuviese dificultad en disponer de todas las rentas que se le habían asignado. Ya en el año 1363, Gonşalvo Eanes, procurador del obispo López de Aguiar, se había visto en la obligación de interponer una demanda contra Leonor Rodríguez —-segunda mujer de Gómez Aras de Pallares - motivada por la negativa de ésta a entregar al obispo de Lugo los bienes que su marido había puesto bajo su control para diversas obras en la ciudad, una de las cuales era - como ya hemos analizadola capilla catedralicia de Santo Domingo ${ }^{28}$. Es éste un ejemplo de las complicaciones que pudieron motivar los cambios acontecidos en los acuerdos de 1388.

Sin embargo, se analiza a partir de este punto la interpretación que se nos presenta con serias garantías de certidumbre para intentar acertar en la correcta lectura y valoración de estos hechos.

\footnotetext{
26 «et se por ventura os ditos beens ou parte deles tevermos aforados, ou eallados, ou en outra maneira prometemos et outorgamos de os librar et tirar das maos et poder de aqueles que os teveren por nosa custa et de os dar et entregar libremente sen contenda a a dita capela et a o capelan et capelaes que dela foren [subsçesive] en nome della». Madrid, A.H.N., Sección Clero (Lugo-Cat.), Carp. $1332 \mathrm{G}$, doc. $\mathrm{n}^{\circ} 12$ (Traslado notarial de 1421-10-01)

27 «Et por mayor seguro de atender et conprir o que sobredito he obligamos especialmente et expresamente a nosa amistraçon domes de janeiro, que ora ten don Diego Gomes, thesoreyro de Lugo, que esta presente et outorgante, con todos seus dereytos et jures et pertenenças, que vos seia por elo obligados enna maneira que dita he, os quaes son da nosa mesa, en tal guisa que non atendendo et comprindo todas estas cousas sobreditas et cada huna delas que o capelan et capelaes que foren suçesive enna dita capela posan entrar et tomar os ditos beens por sua abtoridade sen outra justiça alguna sen enbargo de nos et de nosos suçesores». Madrid, A.H.N., Sección Clero (LugoCat.), Carp. $1332 \mathrm{G}$, doc. $\mathrm{n}^{\circ} 12$ (Traslado notarial de 1421-10-01)

28 Lugo, Archivo Catedral, doc. $n^{\circ} .35$, legajo $n^{\circ} 5$; estante $n^{\circ} 3$. Pergamino en perfecto estado de conservación fechado en el 29 de noviembre de 1363 en el que se refleja el pleito sostenido entre Pedro López de Aguiar y Leonor Rodríguez por el cual la segunda resulta condenada a ceder al obispo los bienes legados por Gómez Aras de Pallares, de los cuales se había apropiado indebidamente y sin realizar el inventario oportuno.
}

Hispania, LXI/2, núm. 208 (2001) 475-492 
Hemos apuntado anteriormente las intenciones del obispo por constituir con su capilla un elemento de enaltecimiento del linaje de los Aguiar y un ejemplo donde se apreciasen los valores de un culto digno, cuidado y magnificente que contase con la dedicación particular de un capellán y con la participación específicamente dispuesta del resto de los capitulares. Para alcanzar estos objetivos don Pedro utiliza los medios económicos que la Mesa Episcopal y las ricas donaciones han puesto en sus manos. Precisamente en razón de esto resulta sorprendente, en la reunión de 1388, la pasividad del prelado ante la actuación del Cabildo en relación con las rentas destinadas a la canonjía en 1379, a no ser que las necesidades económicas requeridas para el cumplimiento de las estipulaciones litúrgicas de la capilla de Santo Domingo quedasen cubiertas con algún ingreso de notoria importancia, cuya naturaleza no aparece explicitada en el acta notarial que se emplea como base documental de nuestra investigación.

La pista para corroborar esta hipótesis nos la proporciona el documento real del día 17 de Abril de 1380. En este documento se solicita al nuevo rey - Juan I- que confirme a la Catedral de Lugo una importante donación de 7.500 maravedíes sobre las rentas del puerto de Viveiro concedida años antes por Enrique II. El monarca manifiesta lo que sigue a continuación:

«que el obispo de Lugo nos embio diser que el rey don Enrique nuestro padre, que Dios perdone, que fiso merced a la iglesia de Lugo et a el et a los beneficiados de la dicha yglesia, de siete mill et quinientos maravedis, que obiessen cada anno en los diesemos de Vivero et que el por mandado del dicho rey nuestro padre que fiso et edifico en la dicha yglesia de los dichos maravedis una capilla en que estan capellanes et dizen en ela missa de cada dia, et ruegan a Dios por el anima del dicho rey nuestro padre et por la nuestra vida et salud et del ynfante mio fijo et de las reynas nuestra madre et mi muger; et que el dicho rey que lles mando pagar los dichos maravedis enteramiente de los primeros e mexor parados $29 \%$.

Sorprende la mención en que se nos informa sobre la orden de construcción emanada del rey Enrique II. No podemos negar taxativamente esta afirmación, sobre todo cuando no es en absoluto improbable que gran parte de esta importante donación se destinase a la construcción de la capilla. Sin embargo, ciertos indicios que ya conocemos, nos muestran que la orden de construcción no provenía del rey Enrique: el hecho de que Gómez Aras de Pallares formule en el documento $^{25}$ de 1357 — citado anteriormente-y en su testamento ${ }^{26}$ del año 1360 unas donaciones para la capilla que se iba a construir en la catedral nos obliga a pensar que, incluso antes de que el propio Enrique II llegase a ser rey, don Pedro López de Aguiar - en esos años aún ferviente colaborador del monarca Pedro I - tenía ya en marcha el proyecto de construcción de su capilla.

29 Madrid, A.H.N., Códice 417 B, folio 8 v.

Hispania, LXI/2, núm. 208 (2001) 475-492 
Por otra parte, el primer documento que nos aclara la celebración de ceremonias litúrgicas en honor de la familia real se fecha en el mes de abril de $1380^{30}$; resulta sorprendente que si la capilla se había concebido por parte de Enrique II y del propio don Pedro López como "capilla real", estas obligaciones litúrgicas que se citan y fijan en 1380, no aparezcan estipuladas en forma alguna en el documento de 1379 donde se concretan los acuerdos con el Cabildo, ritos litúrgicos que - como ya hemos visto- se establecen exclusivamente en provecho de las personas que con sus donaciones contribuyeron a la construcción y dotación de la capilla.

Esto nos obliga a concluir que tenemos ante nosotros un claro intento del obispo por vincular la capilla construida por él con la propia familia real, aprovechando la particular circunstancia del fallecimiento de Enrique II, y pretendiendo justificar una importante merced real con una hipotética orden de construcción por parte del monarca fallecido. Simultáneamente, el prelado hace ver al Cabildo cómo la capilla de Santo Domingo se convierte en justificación de una importante renta que aliviaría las necesidades capitulares, potenciando así la importancia de la capilla y del capellán-canónigo dentro de la estructura interna del conjunto capitular.

Sin embargo, ya se trate de un sutil y admirable ardid del obispo, ya se nos muestre el indicio de una auténtica fundación real - lo que no implica necesariamente la conservación de una orden de construcción -, lo cierto es que la adscripción a las prebendas capitulares de las rentas en metálico provenientes del puerto de Viveiro - que, dicho sea de paso, muy pocas veces llegaron a ser cobradas-, así como el pago de los actos litúrgicos encargados por la familia real, derivados de la nueva condición conseguida por don Pedro para la capilla en cuanto "capilla de reyes", cumplirían largamente el propósito del prelado de otorgar relevancia a su fundación por encima de las otras.

Conseguía paralelamente un honroso y simbólico reconocimiento para la casa de Aguiar, ya que, relacionando su construcción con la familia real, sitúa el escudo de su propio linaje en la entrada de la «capilla real» de la catedral lucense hasta hoy en día.

Por otra parte, todo esto podría posibilitar al obispo la continuación de su labor en favor del Cabildo, al poder concederle permiso para disponer de las rentas que habían constituido el patrimonio inicial de la capilla, aumentando así el de la Mesa Capitular. Lo único que solicita don Pedro es que este cambio de situación quede estipulado por escrito, contando con el firme compromiso del Cabildo de reintegrar las propiedades consabidas a la capilla de Santo Domingo en el caso de que su capellán no fuese admitido en el grupo de los canó-

30 «Sabeam todos que eu Arias Peres, archidiago de Deçon, teente os bees da capella de San Domingo, que noso sennor don frey Pero Lopes obispo de Lugo tornou en esta iglesia de Lugo por tal que se dissesen en ela misas por la alma del rey don Enrrique, a que Deus perdoe, et por la vida del rey don Iohan, que Deus mantenna, et dous outros reys de Castella que despoys del veeren». Madrid, A.H.N., Carp. 1332 G, doc. $n^{\circ} 1$. 
nigos, careciendo entonces de la prebenda pertinente y necesitando de las rentas fijadas en el documento de 1379 para poder garantizar su mantenimiento.

Por lo tanto, y una vez más, observamos, a modo de conclusión, como nuevamente el obispo don Pedro López de Aguiar consigue llevar a buen fin los objetivos fijados sobre la base de los criterios de actuación que hemos analizado a lo largo de este trabajo y que, de una forma en exceso simplificadora, podríamos resumir básicamente en el cuidado y adecuado gobierno de su sede episcopal y en el esfuerzo escrupuloso por mantener su propio linaje como grupo de poder en el Lugo del siglo XIV. En este sentido, a través de la investigación de una práctica de gobierno muy concreta y puntual desarrollada en el ámbito diocesano lucense, se ha reflexionado sobre temas tan generales en el período bajomedieval como el funcionamiento interno de una institución capitular y sus vinculaciones con la autoridad episcopal, sobre las relaciones entre la Iglesia y la Monarquía, o en torno a una temática tan tradicional como las "Mercedes Enriqueñas" generosamente concedidas por la nueva dinastía Trastámara que el obispo don Pedro aprovecha, consiguiendo la confirmación del monarca, para mejorar económicamente la institución capitular, prestigiar al fundador de la orden dominicana y a la propia catedral lucense, alcanzar una situación de privilegio para su grupo familiar y obtener una vinculación particular entre el linaje de Aguiar y la propia familia real. 